\title{
Gravitino production during preheating and the equivalence theorem
}

\author{
A. L. Maroto and J.R. Peláez ${ }^{\text {a* }}$ \\ aDept. Física Teórica, Universidad Complutense de Madrid, 28040 Madrid, Spain
}

We review our results on the calculation of helicity $\pm 1 / 2$ gravitino production during preheating. The method we present is based on the equivalence between goldstinos and longitudinal gravitinos at high energies. The problem is thus reduced to the standard (Majorana) fermion production after inflation. Comparison between helicity $\pm 3 / 2$ and $\pm 1 / 2$ production and with the results obtained in the unitary gauge is also presented.

\section{Introduction}

It is known that global supersymmetry allows the building of inflation models in a relatively easy way. This is mainly due to the presence of scalars fields in the theory and the fact that their potential terms typically contain flat directions. Those flat directions, in addition, are not spoiled by radiative corrections thanks to the non-renormalization theorems. However when dealing with inflationary cosmology, global supersymmetry is not a good description and gravitational effects should be introduced by promoting supersymmetry to a local symmetry, i.e. supergravity. The supergravity corrections to the inflaton potential can have very important effects, spoiling its flatness ( $\eta$ problem). In addition, the appearance in the spectrum of very long-lived particles, such as the gravitino, that couples only with gravitational strength to the rest of matter fields, can pose new problems during nucleosynthesis, since their decay products could destroy the nuclei created in this period. This fact imposes a bound on the abundance of gravitinos given by $n / s<10^{-14}$ for a gravitino mass around $m_{3 / 2} \simeq 1 \mathrm{TeV}$. For this reason, it is very important for the consistency of any model of inflation based on supergravity to keep the gravitino production under control.

Gravitinos can be created by particle collisions in the thermal bath that appears after inflation. The corresponding calculations of the particle

\footnotetext{
*This work has been partially supported by CICYTAEN97-1693, PB-98-0782 and Ministerio de Educación y Ciencia (Spain).
}

production can be done by standard perturbative techniques and leads to the well-known limit on the reheating temperature $T_{R}<10^{9} \mathrm{GeV}$, for gravitinos with mass around $1 \mathrm{TeV}$. However, gravitinos could also be created directly from the inflaton field during the period of its coherent oscillations after inflation, provided the inflaton field couples directly to the gravitino [1,22. Under these conditions, and during the first stages when the amplitude of the oscillations is large, the perturbative technique is no longer valid, and different non-perturbative approaches, such as Bogoliubov transformations or lattice simulations, are required.

In this note we review our results on a new technique for the calculation of the helicity $1 / 2$ gravitino production, based on the combination of Bogoliubov transformations and the high-energy equivalence between goldstinos and longitudinal gravitinos [3].

\section{Gravitino helicities and the equivalence theorem}

Gravitinos are the spin 3/2 supersymmetric partners of the graviton field. As long as supersymmetry remains unbroken, the gravitino is a massless field like the graviton and, as a consequence, it can only appear in two possible helicity states, namely, $\pm 3 / 2$. However, when supersymmetry is spontaneously broken, the gravitino becomes a massive field and then the $\pm 1 / 2$ helicities are also possible. This is nothing but the supersymmetric version of the Higgs mechanism, although, instead of a pseudoscalar mass- 
less Goldstone boson "eaten" by a gauge boson that acquires a longitudinal component, we now find a Majorana massless fermion, or goldstino, "eaten" by the gravitino to acquire $\pm 1 / 2$ helicity components.

Intuitively, then, one is tempted to identify the $\pm 1 / 2$ helicity gravitino components with those of the goldstino in a global supersymmetric theory. However, since those goldstinos are massless and gravitinos are not, this intuitive identification can only be carried out at energies high enough for the gravitino mass, $m$, to be negligible. The rigorous formulation of this statement is known as the goldstino-gravitino Equivalence Theorem. The use of this theorem results in a major simplification, since it is far simpler to work with Majorana fermions than with the Rarita-Schwinger spin 3/2 massive gravitino projected along the $\pm 1 / 2 \mathrm{com}-$ ponent.

Why $\pm 1 / 2$ gravitinos are so relevant for preheating? The reason is that the production of gravitinos with $\pm 1 / 2$ helicities is, in general, dominant during preheating. This is due to the fact that we expect the physical momentum of the gravitinos, $p_{\mu}$, to be of the order of the inflaton mass, $m_{\phi}$, which, typically, is many orders of magnitude larger than the gravitino mass. If we now look at the expression for the $\pm 1 / 2$ helicity projectors, we find that they behave as $P_{\mu}^{ \pm 1 / 2} \sim p_{\mu} / m+O(m / p)$, i.e., they grow with the momentum. In contrast, the $\pm 3 / 2$ projectors behave just like a constant of order one. Consequently, if we want to calculate the number of gravitinos created after inflation, we are basically dealing only with very energetic $\pm 1 / 2$ gravitinos. Fortunately, with the Equivalence Theorem are much easier to handle since they can be identified with the Majorana goldstinos.

\subsection{Sketch of the proof}

The proof of the Equivalence Theorem, is somewhat technical, and we refer to our work for mathematical details [3]. Our purpose here is to sketch the main ideas involved in its derivation and review the final result as well as the applicability conditions. Let us consider the fermionic part of the minimal supergravity Lagrangian with a single chiral superfield which contains an scalar field $\phi$ (inflaton) and a Majorana spinor $\eta$ (inflatino, goldstino):

$$
\begin{aligned}
g^{-1 / 2} \mathcal{L}_{F} & =-\frac{1}{2} \epsilon^{\mu \nu \rho \sigma} \bar{\psi}_{\mu} \gamma_{5} \gamma_{\nu} D_{\rho} \psi_{\sigma} \\
& +\frac{i}{2} \bar{\eta} \not D \eta+e^{G / 2}\left(\frac{i}{2} \bar{\psi}_{\mu} \sigma_{\mu \nu} \psi^{\nu}\right. \\
& +\frac{1}{2}\left(-G_{, \phi \phi}-G_{, \phi}^{2}\right) \bar{\eta} \eta \\
& \left.+\frac{i}{\sqrt{2}} G_{, \phi} \bar{\psi}_{\mu} \gamma^{\mu} \eta\right)+\frac{1}{\sqrt{2}} \bar{\psi}_{\mu}(\not \partial \phi) \gamma^{\mu} \eta
\end{aligned}
$$

where the Kähler potential is given by $G\left(\Phi, \Phi^{\dagger}\right)=$ $\Phi^{\dagger} \Phi+\log |W|^{2}$. Note that the last two terms mix the gravitino, $\psi_{\mu}$, and goldstino fields, and therefore their equations of motion are coupled.

Let us then recall that supergravity is a gauge invariant theory, invariant under local supersymmetry transformations. Nevertheless, in order to make a specific calculation it is necessary to choose a gauge, although the final result for the observables will be independent of this choice. For instance, we could choose the unitary gauge, where we gauge away the goldstinos and make explicit the mass of the gravitino. In such a case we would have to deal with the four helicities of the gravitino spin $3 / 2$ field and therefore with the complicated $\pm 1 / 2$ helicity projectors [2]. But in any other gauge the goldstinos will still be present in the Lagrangian, although there will be a mixing kinetic term between goldstinos and the derivative of the gravitinos. This is the kind of gauges where the Equivalence Theorem can be derived 顿. Indeed, in these gauges, known as t'Hooft or $R_{\xi}$ gauges, the gauge fixing function relates the goldstino to the derivative of the gravitino field over its mass. The explicit gauge-fixing condition is given by:

$\gamma^{\mu} \psi_{\mu}-\frac{1}{\sqrt{2} \xi \not D} e^{G / 2} G_{, \phi} \eta+\frac{i e^{-G / 2}}{G_{, \phi}} \gamma^{\mu}(\not \partial \phi) \psi_{\mu}=0$

that can be written asymptotically (before and after preheating) as:

$\partial^{\mu} \psi_{\mu}=\sqrt{\frac{3}{2}} \frac{m}{\xi}\left(1-\xi \frac{m_{ \pm}}{m}\right) \eta$.

Indeed, the derivative of the gravitino field is proportional to the goldstino, with a proportionality 
constant that depends on $\xi$. Since, in momentum space, the derivative becomes the gravitino momentum, and we have just seen that the $\pm 1 / 2$ helicity projector $P_{\mu}^{ \pm 1 / 2}$ behaves precisely as the momentum over the mass, we find that, in those gauges, the goldstinos are nothing but the $\pm 1 / 2$ gravitinos, whenever $p \gg m$. That is the standard proof of the Equivalence Theorem.

When dealing with the inflationary supergravity scenario, there are some subtleties in this proof that we have addressed in our work 33. Among others, it is specially relevant the fact that we need to establish the equivalence in terms of classical equations of motion in order to apply the Bogoliubov transformations. However, previous proofs were given in terms of a path integral formulation or in the context of S-matrix theory [4]. Further complications come from the definition of the asymptotic initial and final states, the introduction of classical sources like curvature and the inflaton itself, the fact that due to the expansion the initial and final momenta are scaled with the Universe scale factor, etc... We refer to our work for the details.

The important result is that in the asymptotic initial and final states, the classical solutions of the goldstino equations are proportional to those of the $\pm 1 / 2$ helicity gravitinos. Since the proportionality constant is irrelevant to calculate the Bogoliubov coefficients (because they satisfy a normalization constraint), the production of $\pm 1 / 2$ gravitinos can be read directly from the calculation using just goldstinos.

\subsection{Applicability conditions}

The previous statement can be applied only under the following conditions:

- The frequency of the inflaton oscillations should be larger than the gravitino mass $m_{\phi} \gg m$. If we are interested in pure gravitational production one should also require $H \gg m$.

- The classical sources should vanish asymptotically, which implies that both the space time curvature as well as the inflaton oscillations should decrease with time.
- Since we are dealing with ensembles of gravitinos with a non-thermal distribution, we have to be sure that the vast majority of the gravitinos are energetic enough, which implies $a_{\text {out }} m \ll a_{\text {in }} m_{\phi}$, where $a_{\text {in }}$ and $a_{\text {out }}$ are the Universe scale factor before and after preheating, respectively.

The first, which we had already met, ensures that the typical gravitino momentum is larger than its mass. The second is needed for the definition of particles in the asymptotic regions. Concerning the third, the fact that there is an exponential growth of the scale factor during inflation may seem to jeopardize the applicability of the Theorem. However, preheating only takes place during the first few inflaton oscillations, so that the scale factor only grows by a few orders of magnitude, in contrast with the many orders of magnitude between $m$ and $m_{\phi}$, so that the Equivalence Theorem can be safely applied.

It is important to note that, although the $\pm 1 / 2$ helicity gravitinos and the goldstinos may evolve differently during the inflaton oscillations, they tend to each other in the asymptotic regions (up to a proportionality constant).

\subsection{The calculation with goldstinos and the Landau gauge}

Once we have established that we simply need to study the gravitino production in terms of the much simpler goldstino fields, next, we have to calculate the evolution of goldstino plane waves under the goldstino equations of motion, and compare the particle number in the initial and final states.

The problem is that even though we are simply looking at Majorana fermions, their equations of motion are still rather complicated in a general $R_{\xi}$ gauge. From eq.(11) we obtain:

$$
\begin{aligned}
i \not D \eta- & e^{G / 2}\left(G_{, \phi \phi}+G_{, \phi}^{2}\right) \eta \\
& -e^{G / 2} G_{, \phi} \frac{i}{2 \xi \not D} e^{G / 2} G_{, \phi} \eta=0
\end{aligned}
$$

However, we still have the freedom to choose the gauge fixing variable $\xi$ to obtain a further simplification. The most convenient choice seems to be $\xi \rightarrow \infty$, known as the Landau gauge, since then 
the goldstinos obey a well known Dirac-like equation, which can be easily integrated numerically.

\section{Numerical example with a toy model}

In order to illustrate the above analysis we will consider a particular example with a superpotential given by:

$W=\sqrt{\lambda} \frac{\Phi^{3}}{3}$,

According to the Equivalence Theorem, the number of helicity $\pm 1 / 2$ gravitinos created by the oscillating inflaton can be obtained by solving the equation for the Fourier modes of the goldstinos coming from (1). Using the standard Bogoliubov coefficients technique we obtain the results for the spectrum shown in Fig.1. (The helicity $\pm 3 / 2$ results have been obtained following the analysis in [1]). We see that for this particular model,

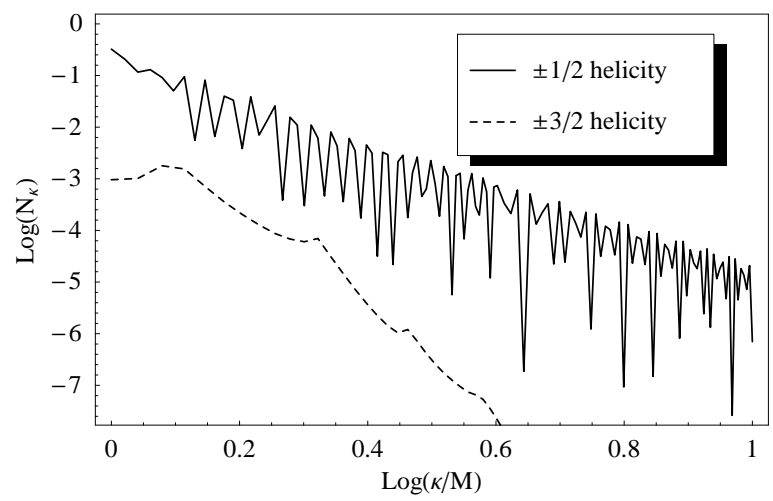

Figure 1. Spectrum of helicity $\pm 3 / 2$ and helicity $\pm 1 / 2$ gravitinos. The helicity $\pm 1 / 2$ production has been obtained using the ET.

the production of helicity $\pm 3 / 2$ gravitinos is suppressed by two to three orders of magnitude with respect to the $\pm 1 / 2$ gravitinos. Integrating the spectrum over the whole range of momenta we obtain the total number of gravitinos created during preheating. For the helicity $\pm 1 / 2$ we obtain $n / s \simeq 10^{-10}$. Comparing this result with the nucleosynthesis bounds we observe that this model would be ruled out if the gravitino mass is around (or smaller than) $1 \mathrm{TeV}$. We have found a very good agreement between these results and those obtained in the unitary gauge [2] for the same model.

\section{Future and Possible extensions}

In the previous discussion we have concentrated on the simplest case in which we have minimal supergravity and only one chiral superfield. We should recall that apart from a successful inflationary period, we also require from our model that supersymmetry is broken at the minimum of the potential, otherwise the production of helicity $\pm 1 / 2$ gravitinos would be meaningless. Precisely for this reason, the single-field theories cannot be considered, in general, as realistic models for supergravity inflation since, on the one hand, they typically exhibit the $\eta$ problem described in the introduction, and, on the other hand, it is extremely difficult to find an appropriate superpotential that breaks supersymmetry at the minimum and, at the same time, contains the appropriate inflationary scale. It is then very interesting to extend the analysis to the multi-chiral fields models in which it is possible to accommodate these requirements $[5]$. In this case, although the identification of the goldstino is not straightforward and in general it will be a timedependent combination of fermionic fields, we expect on physical grounds an extended version of the Equivalence Theorem will also hold. Work is in progress in this direction.

\section{REFERENCES}

1. A.L. Maroto and A. Mazumdar, Phys. Rev. Lett. 841655 (2000)

2. R. Kallosh, L. Kofman, A. Linde and A. Van Proeyen,Phys. Rev. D61 (2000) 103503; G.F. Giudice, A. Riotto and I. Tkachev, JHEP (1999) 9911:036 and hep-ph/9911302

3. A.L. Maroto and J.R. Peláez, Phys. Rev.D62 (2000) 023518

4. R. Casalbuoni, S. De Curtis, D. Dominici, F. Feruglio and R. Gatto, Phys. Lett. B215, 313 (1988) and Phys. Rev. D39, 2281 (1989).

5. R. Kallosh, L. Kofman, A. Linde and A. Van Proeyen, Class.Quant.Grav 17 (2000) 4269 